\title{
Prototipo de un generador eólico doméstico con materiales asequibles en El Salvador
}

\section{Carlos Roberto Martínez Martínez}

Docente, Facultad de Ingeniería y Arquitectura

Universidad Católica de El Salvador

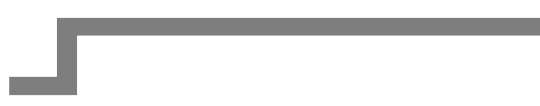

Recepción: 2014-01-08

Resumen

Aceptación: 2014-02-10

Este trabajo de investigación tiene como resultado el diseño y construcción de una Aero turbina de eje vertical de bajo costo, a fin de verificar la factibilidad técnica y económica de incursión de este prototipo en aplicaciones de generación de energía eólica a pequeña escala en El Salvador.

Se crearon diferentes modelos de turbinas para comparar su eficiencia, siendo el más exitoso el rotor de eje vertical con cuatro aspas semicirculares; el cual es capaz de operar a partir de vientos suaves de $5 \mathrm{Km} / \mathrm{h}$. Por otra parte, también se experimentó con generadores de corriente alterna y directa, en busca de la mejor relación entre costo económico y producción energética. Durante el proceso se pusieron a prueba varios conceptos relacionados con el tema, y que son populares en el Internet. Esto concluyó en la desmitificación de la viabilidad de los alternadores, los cuales son modificados al añadirles imanes circulares permanentes.

Los resultados también demostraron que los motores de corriente directa sí pueden ser configurados para generar la energía suficiente como para iluminar una vivienda pequeña, mediante la utilización de focos tipo led. Sin embargo, su factibilidad también se vuelve cuestionable, debido al cambio climático que acontece a nivel global y que causa condiciones de poco viento en El Salvador; obstaculizándose la generación eólica.

Palabras clave: Energía eólica, turbina, corriente alterna, bajo costo, generadores

\begin{abstract}
This research work has a result the design and construction of an aero turbine with a low cost vertical axis with the purpose of check the technical and economic feasibility of the implementation of this prototype in applications of generating eolian energy on a small scale in El Salvador.

Different models of turbines were created to compare their efficiency, being the most successful one the rotor with vertical axis one with four semicircular blades that are able to work from $5 \mathrm{~km} / \mathrm{h}$ gentle winds. On the other hand, it was also experimented with generators of alternating or direct current in order to look for the best relationship among the economic cost and the energetic production. During the process, some concepts related to the topic were tested as they are popular in the internet. This concluded to demystify the variability of the alternators that were modified by adding permanent circular magnets of them.
\end{abstract}

The results also showed that the direct current motors can be set up to generate enough energy to illuminate a small house by using LED spotlights. However, its feasibility can also be questioned due the weather change happening in the world that causes little wind in El Salvador blocking the eolian generation

Key words: Eolian energy, turbine alternating current, low cost, generators 


\section{Introducción}

Existen diversas implementaciones de energías alternativas orientadas al servicio residencial. Entre algunos de sus beneficios se encuentran: aminorar el impacto al medio ambiente, disminuir costos por electricidad, o simplemente proveer el servicio a comunidades que están alejadas de la red comercial.

Esta investigación incursionó en el campo de la generación de energía eólica, mediante el diseño de una turbina que se accionara por acción del viento, transmitiendo la suficiente fuerza mecánica para activar un generador eléctrico. Se buscó que este fuera un mecanismo de bajo costo, capaz de proporcionar iluminación a una vivienda pequeña, en condiciones de viento moderado; al mismo tiempo que su fabricación fuera posible, a partir de materiales disponibles en el mercado local.

No obstante, existe la limitación de que en El Salvador no se dispone de zonas en donde las velocidades de viento sean altas ${ }^{1}$. De acuerdo a datos recopilados, los mayores promedios de viento se encuentran en la parte nortecentral del país, con valores entre los 18 a 22 $\mathrm{Km} / \mathrm{h}$. El resto del territorio tiene velocidades promedio entre los 14 a $18 \mathrm{Km} / \mathrm{h}$, con un patrón de desplazamiento más o menos constante a lo largo del año.

1. MARN y MAGMA/UCA (2005).
En virtud de estos hechos, el diseño del prototipo de energía eólica se orientó hacia la funcionalidad, específicamente en condiciones de vientos leves a moderados.

\section{Materiales y Métodos}

En primer término, se crearon tres modelos diferentes de aero turbinas a escala, las cuales se apoyaron en simulaciones asistidas por computador. Estos prototipos fueron probados en un túnel de viento bajo condiciones ideales - para luego ser contrastados en a campo abierto y determinar cuál de ellas poseía mejor desempeño. Luego de las pruebas pertinentes, se optó por el prototipo que reunía las condiciones de mayor eficiencia y viabilidad económica, en cuanto al costo de los materiales utilizados en su fabricación y su construcción. Luego de construirse a escala real el prototipo seleccionado, este se instaló en campo abierto para realizar el conteo de revoluciones por minuto. Se le acoplaron embudos de viento a su alrededor para inducir un aumento en la velocidad de la ráfaga.

Aparte de ello, se experimentó con diferentes tiposdegeneradores eléctricos quecumplieran el mismo requisito primordial de ser de bajo costo. Al igual que con el prototipo, con los generadores se determinaron sus respectivas ventajas y limitaciones, tras la realización de pruebas de producción de corriente alterna y corriente continua. Dichas pruebas se 
realizaron con el fin de medir la relación entre la velocidad del viento, la cantidad de rotaciones realizadas por la turbina y la potencia de la energía generada.

Finalmente, se implementó una transmisión que permitiría aumentar la velocidad de rotación del sistema completo, conectando el eje de la turbina como origen del movimiento mecánico hacia el generador eléctrico.

\section{Resultados y Discusión}

\subsection{Diseño de la aeroturbina}

Se construyeron modelos a escala de tres tipos de turbinas: la primera se creó con base en el modelo clásico de hélice; la segunda, basada en el modelo de tres alerones, copiando la silueta transversal del ala de un avión; y la tercera, de acuerdo a un modelo de aspas semicirculares.

En cada tipo se incluyó el flujo aerodinámico. Para los dos primeros modelos, cada aspa aporta su propio empuje al sistema según un comportamiento aerodinámico independiente. En cambio, para el tercer modelo, el aire que impacta en un aspa semicircular se desplaza incrementando el flujo en las demás, creando así un efecto sinérgico (Ver figuras 1-3).

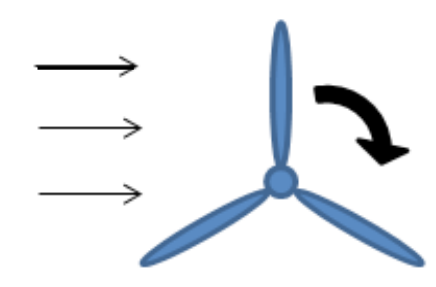

Figura 1. Modelo de hélice vertical (2014).

Las flechas delgadas de la izquierda - que muestran un desplazamiento hacia la derecha - simulan la dirección de entrada del viento; mientras que la flecha en negrita - que muestra un sentido de la rotación acorde a las agujas del reloj - denota la trayectoria que deben seguir las aspas como resultado de la acción del viento.

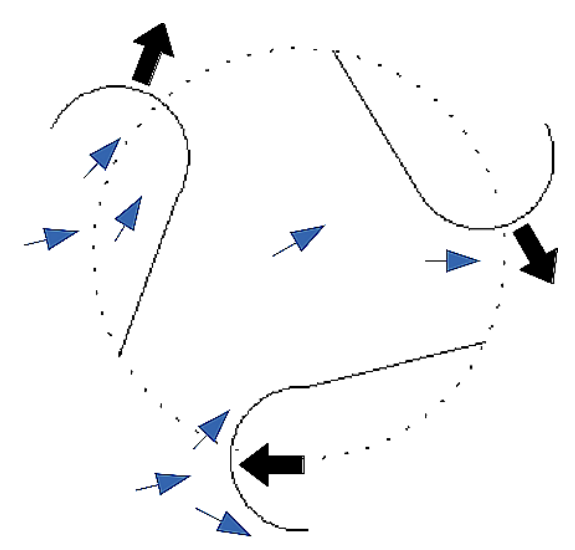

Figura 2. Modelo de alerones huecos (2014).

En el segundo modelo, las flechas pequeñas muestran las distintas y posibles trayectorias de entrada del viento, así como también dentro de la turbina; mientras que las flechas en negrita muestran los movimientos circulares que deben seguir las aspas ante la acción del viento. 


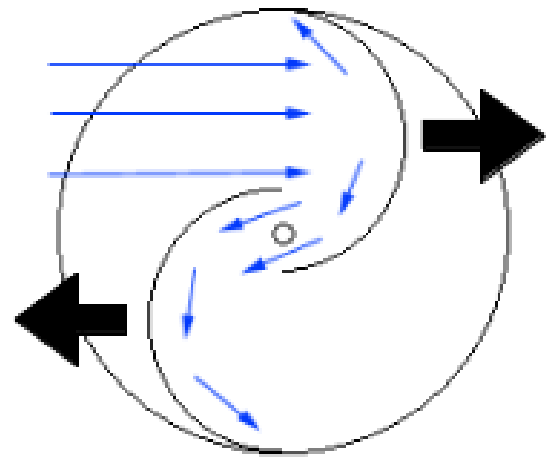

Figura 3. Modelo de aspas semicirculares (2014).

Al igual que en primer modelo, las flechas delgadas, que señalan un desplazamiento hacia la derecha, describen el ingreso del viento hacia la turbina. Una vez dentro, el aire (flechas delgadas y cortas) se filtra en dos direcciones: hacia arriba y hacia abajo, de acuerdo al diseño de las aspas de la turbina. Por su parte, las aspas se mueven únicamente en dos direcciones, según lo indican las flechas en negrita (Figura 3).

Cada modelo fue probado en forma independiente, mediante su instalación dentro de un túnel, con vientos a velocidades de $5 \mathrm{Km} / \mathrm{h}$ y $40 \mathrm{Km} / \mathrm{h}$.

De acuerdo con los experimentos realizados con los tres diferentes modelos, la opción de aspas circulares es la que mejor aprovecha la energía del flujo de aire, tanto a baja como alta velocidad (Tabla 1). Esto resultó muy conveniente, pues este modelo fue el más fácil de fabricar.

Tabla 1. Pruebas de rotación de cada modelo de turbina a baja y alta velocidad de viento

\begin{tabular}{|c|c|c|}
\hline Modelo a escala & $\begin{array}{c}\text { Velocidad de } \\
\text { viento: } \mathbf{5} \mathbf{~ K m} / \mathbf{h}\end{array}$ & $\begin{array}{c}\text { Velocidad de viento: } \\
\mathbf{4 0 ~ K m} / \mathbf{h}\end{array}$ \\
\hline Hélice vertical & $0 \mathrm{rpm}^{2}$ & $60 \mathrm{rpm}$ \\
Alerones huecos & $8 \mathrm{rpm}$ & $64 \mathrm{rpm}$ \\
Aspas semicirculares & $14 \mathrm{rpm}$ & $115 \mathrm{rpm}$ \\
\hline
\end{tabular}

Nota: Se muestran los resultados obtenidos bajo condiciones controladas de estudio.

Una vez seleccionado el tipo de turbina a La energía de las rotaciones se condujo a utilizar, se procedió a elaborar el diseño través de una transmisión de poleas, que completo del generador, el cual constituyó incrementaban las revoluciones por minuto de la turbina misma sujetada a un mástil, del generador. Este último producía la mediante baleros que permitieran su rotación. electricidad suficiente para ser utilizada Estos, por su parte, debían estar acoplados a directamente; o para ser almacenada en una una base fija que diera soporte a las fuerzas de la turbulencia y vibraciones provocadas por los vientos (ver figura 4). batería para su posterior uso. Para que todo este sistema fuera funcional, el flujo eléctrico se dirigió en una sola dirección - desde el 
generador hacia la batería y no viceversa - ya que eso podía causar gastos indeseables de energía. Además, la corriente no debía tener una intensidad estable, debido a que el viento tampoco se manifiesta de esa forma. Por ello, se integró un circuito regulador que permitió controlar los parámetros normales de recarga de la batería. Este último componente funcionó como fuente de electricidad.

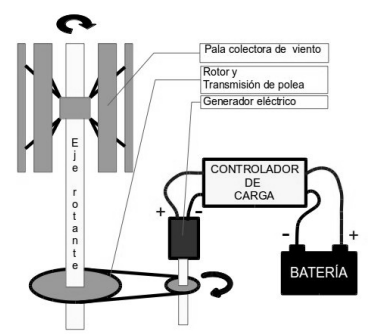

Figura 4. Esquema conceptual del generador eólico (2014).

Mientras las turbinas de hélice horizontal necesitan ser dotadas de un rotor adicional que las oriente hacia la dirección de ataque del viento, el modelo propuesto utiliza una turbina con un rotor de eje vertical, capaz de operar sin importar la dirección de donde venga el viento. Es decir, el modelo planteado es capaz de operar de igual manera sin importar de dónde provenga la ráfaga que lo acciona o qué tan distorsionado sea su flujo. Esto simplifica su construcción, ya que no hace falta incorporar mecanismos adicionales de articulación. Además, otra ventaja sustancial que se comprobó durante la experimentación fue que, la acción de esta turbina es totalmente silenciosa.
Con respecto a la construcción, se utilizó un tubo de hierro de dos pulgadas como eje principal. En él se soldaron los soportes para sujetar las cuatro aspas (figura 5).

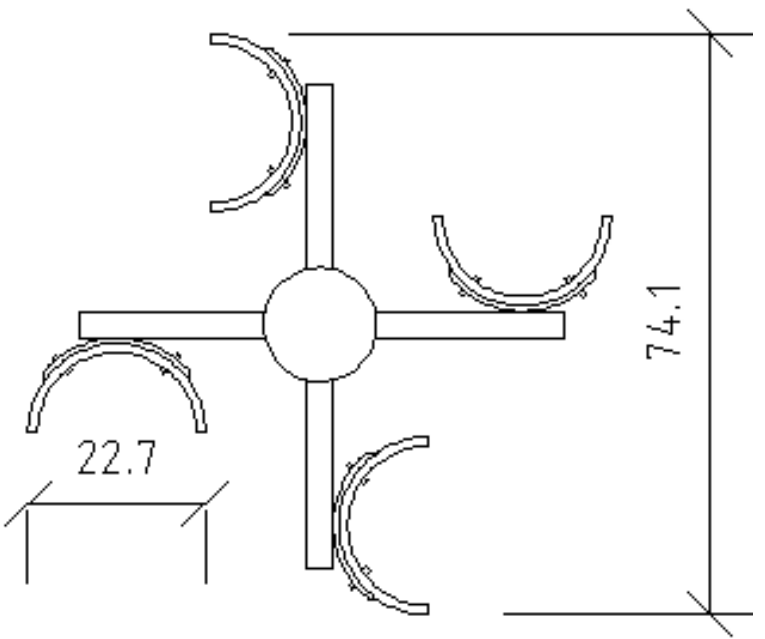

Figura 5. Vista superior del diseño de aeroturbina. Las medidas son expresadas en centímetros (2014).

Las palas utilizadas para la turbina se fabricaron a partir de tubos PVC de 8 pulgadas, con una longitud de metro y medio cada una. para obtener canales semicirculares, los tubos se cortaron de longitudinalmente, debido a que su curvatura generaba un efecto de sustentación similar a las alas de los aviones; específicamente, cuando el viento pasa sobre la superficie curva exterior, aumenta su velocidad y la presión atmosférica disminuye sobre dicha área. En cambio, en la parte interna de las alas, el viento se encierra y circula lentamente, generándose una alta presión. La diferencia entre las presiones, alta en el interior y baja en el exterior, hace que la pala obtenga una 
fuerza de empuje hacia afuera, haciendo rotar la turbina.

Así como sucediera en el modelo a escala, las palas en conjunto tuvieron un efecto sinérgico, ya que las corrientes de aire que afectaban a cada una de ellas, se deslizaron y agregaron a las corrientes de sus vecinas, incrementándose la eficiencia.
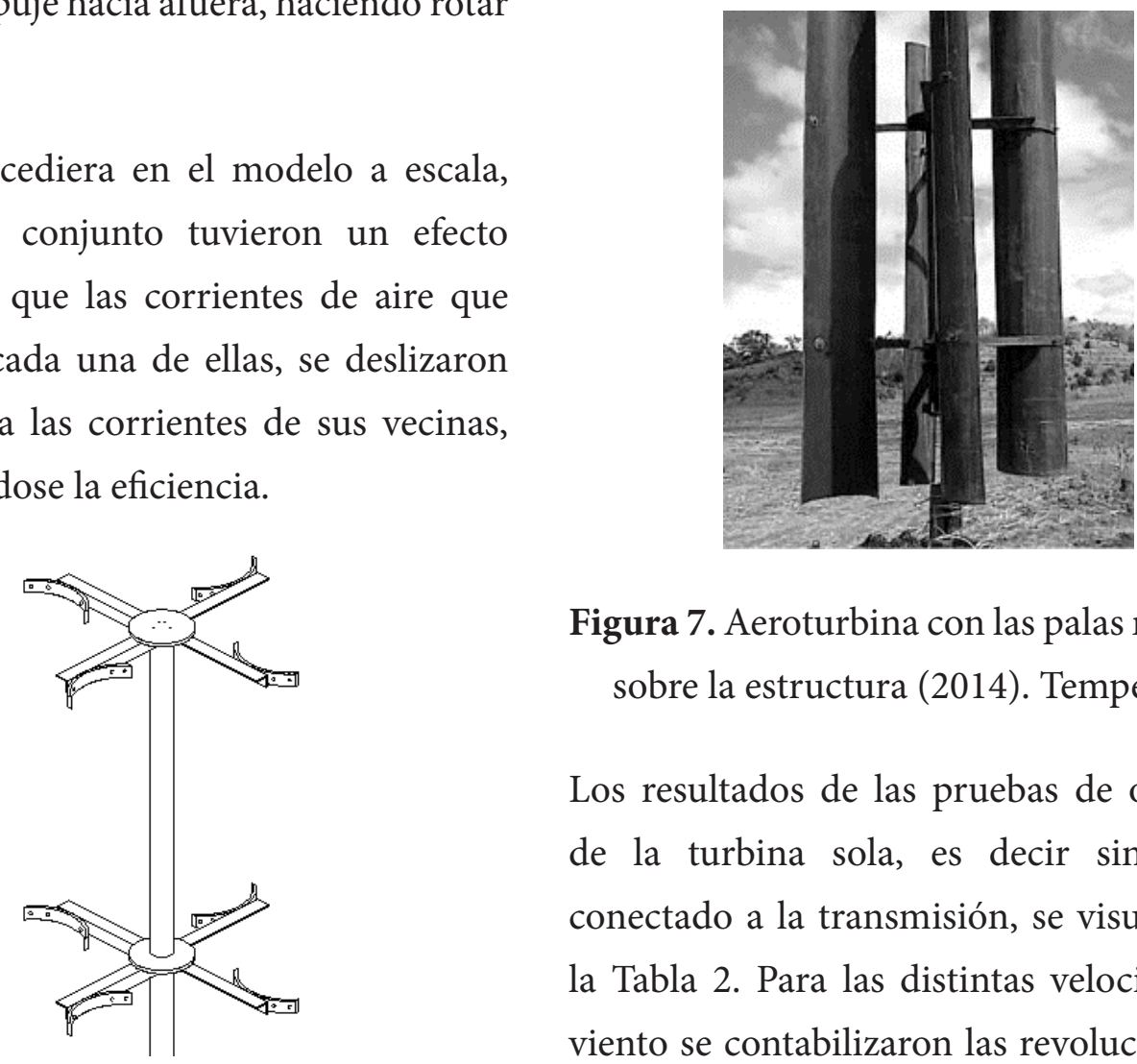

Figura 7. Aeroturbina con las palas montadas sobre la estructura (2014). Temperatura

Los resultados de las pruebas de operación de la turbina sola, es decir sin haberla conectado a la transmisión, se visualizan en la Tabla 2. Para las distintas velocidades de viento se contabilizaron las revoluciones por

Figura 6. Diseño del rotor de la aeroturbina (2014). minuto que fueron producidas. Ante esto se obtuvo una proporción matemática de tipo exponencial (ver gráfica 1 ).

Tabla 2. Revoluciones por minuto (rpm) obtenidas en pruebas de rotación

\begin{tabular}{|c|c|}
\hline Viento $(\mathbf{K m} / \mathbf{h})$ & RPM $^{3}$ \\
\hline 5 & 75 \\
\hline 20 & 107 \\
\hline 30 & 219 \\
\hline 40 & 416 \\
\hline
\end{tabular}

Nota: Se observan los datos obtenidos durante pruebas de rotación a campo abierto.

(3) Entiéndase como revoluciones por minuto. 


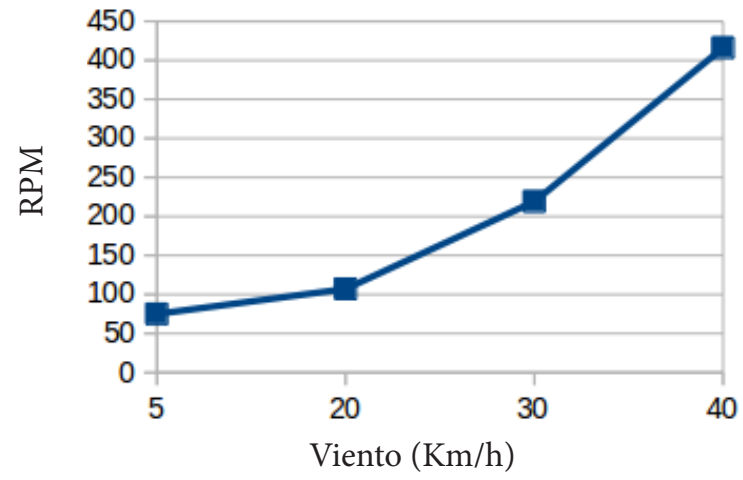

Figura 8. Gráfica pruebas de rotación de turbina. Velocidad del viento vs. revoluciones por minuto

Velocidades de viento por debajo de los 3 $\mathrm{Km} / \mathrm{h}$, no fueron capaces de vencer la fricción interna de los baleros del rotor. Sin embargo, esa intensidad de viento puede considerarse bastante baja.

A partir de los $3 \mathrm{Km} / \mathrm{h}$ se observaron algunas rotaciones no uniformes; pero fue hasta los 5 $\mathrm{Km}$ /que pudo alcanzarse un comportamiento rotacional estable y apto para comenzar a producir electricidad. La relación entre la velocidad del viento y las revoluciones por minuto que experimentó la turbina, fueron modelados a través de la ecuación de regresión

$$
f(x)=17+5.4 e^{\frac{x}{12.3}}
$$

Figura 9. Fórmula para ecuación de regresión, utilizada para medir velocidad del viento y revoluciones por minuto.

\subsection{Diseño del colector de viento}

En teoría, un colector de viento es un conjunto de paletas que rodean una turbina, con el fin de actuar como un embudo que recoge mayor cantidad de flujo, incrementando así las revoluciones del viento.

En la figura 10 se muestran los tres tipos de colectores con los cuales se experimentó: el primero (A) de paletas rectas $u$ ortogonales a la tangente circular; el segundo (B) de paletas tangenciales, y el tercero (C) de paletas curvas.

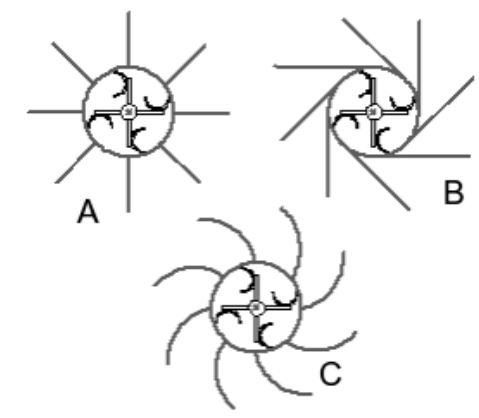

Figura 10. Distintos colectores de viento: recto (A), tangencial (B) y curvo (C) (2014).

En la siguiente tabla se muestra el porcentaje de incremento de rotaciones por cada colector instalado. El colector $\mathrm{C}$ es el que mejor funcionó, debido a la generación del efecto Bernoulli, que sucede cuando el viento recorre una trayectoria curva e induce un incremento en su velocidad.

Durante el desarrollo del estudio, todos los modelos utilizados indujeron solo una leve mejoría en el rendimiento de la turbina, lo cual desestimó su factibilidad. 
Tabla 3. Rendimiento de rotaciones en turbina

\begin{tabular}{|c|c|}
\hline Tipo de colector & Porcentaje \\
\hline Colector recto & $9 \%$ \\
\hline Colector tangencial & $11 \%$ \\
\hline Colector curvo & $15 \%$ \\
\hline
\end{tabular}

Nota: El porcentaje incrementó de acuerdo al tipo de colector de viento instalado en la turbina.

Aunque el colector curvo ofreció mejores resultados en cuanto a rendimiento, se consideró que el beneficio aportado no era significativo como para que se integrara al diseño. Su costo de producción se estimó en $\$ 180.00$ dólares, representando un $30 \%$ del costo total del sistema completo. $\mathrm{Al}$ analizar esa información con su rendimiento, el colector $\mathrm{C}$ solamente proporciona un incremento de $15 \%$ en las revoluciones por minuto.

Por otra parte, al ser el colector un artefacto rígido y de volumen relativamente grande, se encuentra bastante susceptible a sufrir daños por la turbulencia del viento. Esto implica que debe estar muy bien reforzado al momento de su construcción, pues de lo contrario corre el riesgo de que, tarde o temprano acabe por desprenderse.

\subsection{Diseño de la trasmisión}

Existen tres tipos de transmisiones: por poleas, por fricción o por engranes. Las transmisiones por fricción son susceptibles a desgaste y deslizamientos a causa de la humedad, mientras que las transmisiones por engrane necesitan estar dentro de un depósito sellado que contenga aceite para prevenir su desgaste.

Por motivos prácticos, fue más sencillo optar por una transmisión de poleas, haciendo uso de fajas para interconectarlas. Si bien el objetivo de la transmisión era incrementar la cantidad de revoluciones por minuto del sistema completo, esto actuaba en detrimento de la potencia. Es decir, al incorporar una transmisión, se necesitaba de una mayor velocidad de viento para operar la turbina.

La figura 11 muestra el diseño de una transmisión de tres pasos, basado en cuatro poleas, las cuales generan un incremento significativo en las rotaciones. La polea A de 8 pulgadas; la $\mathrm{B}$ de 3 pulgadas, la $\mathrm{C}$ (solidaria al eje de la B) de 5 pulgadas y la D de 3 pulgadas. 


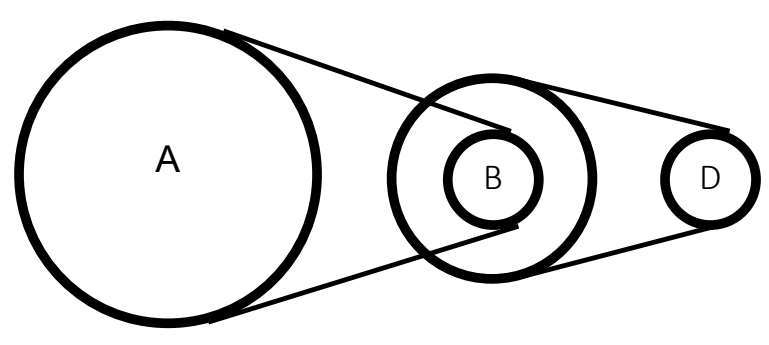

Figura 11. Transmisión de tres pasos. Sistema de cuatro poleas con diferentes diámetros (2014).

Para entender el funcionamiento de este sistema, es necesario mostrar la ecuación que rige la trasferencia de energía:

$$
\mathrm{D}_{\mathrm{A}} \mathrm{V}_{\mathrm{A}}=\mathrm{D}_{\mathrm{B}} \mathrm{V}_{\mathrm{B}}=\mathrm{D}_{\mathrm{C}} \mathrm{V}_{\mathrm{C}}=\mathrm{D}_{\mathrm{D}} \mathrm{V}_{\mathrm{D}}
$$

Donde $D$ simboliza el diámetro de la polea, y $V$ su velocidad de rotación. Para las poleas solidarias $B$ y $C$, su velocidad de rotación es la misma, por lo cual: $\mathrm{V}_{\mathrm{B}}=\mathrm{V}_{\mathrm{C}}$ Por otra parte, la ecuación utilizada para determinar la ventaja mecánica $\mathrm{V}$ en términos de potencia es:

$$
\mathrm{V}=\left(\mathrm{D}_{\mathrm{B}} / \mathrm{D}_{\mathrm{A}}\right)\left(\mathrm{D}_{\mathrm{D}} / \mathrm{D}_{\mathrm{C}}\right)
$$

De acuerdo a los cálculos, el incremento en las rotaciones para esta transmisión fue del $444 \%$, pero la pérdida de potencia fue del $77.5 \%$. Durante la experimentación se comprobó que solo los vientos fuertes podían accionar el sistema mecánico.

La figura 12 esquematiza una trasmisión más sencilla. Este es un modelo de dos pasos que utiliza una polea de 8 pulgadas y otra de 3 pulgadas para funcionar. De acuerdo a los cálculos, el incremento en las rotaciones para esta transmisión fue del 267\%, que equivale aproximadamente a la mitad del porcentaje obtenido en la transmisión anterior. Sin embargo, la pérdida de potencia fue del $37.5 \%$, lo que demostró que la turbina necesitaba vientos de menor intensidad para poder operar.

Durante la experimentación se observó que las revoluciones generadas por la turbina, cuando no estaba conectada a esta segunda

transmisión, eran exactamente las mismas que cuando se encontraba conectada a ella. Esto demostró que no existía un incremento significativo en la productividad.

Al realizarse cálculos posteriores, se demostró que la energía generada al efectuar mayores revoluciones, era igual a la energía que se requería para accionar dicha transmisión. Por lo tanto, la pérdida de potencia representaba un equilibrio con respecto a la ganancia de rotaciones, causando que no hubiese aventajamiento mecánico.

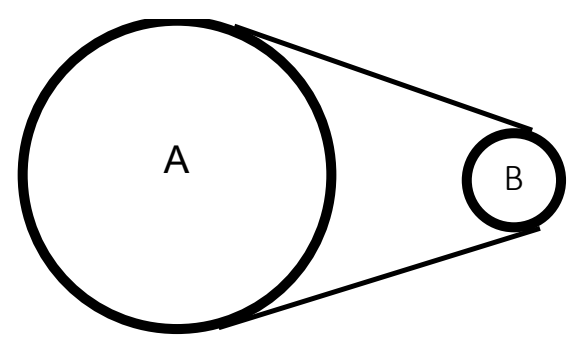

Figura 12. Transmisión de dos pasos. Sistemas dos poleas con diferentes diámetros (2014). 
La tabla 4 resume los resultados de la experimentación con ambas transmisiones.

En cuanto a la primera, si bien el incremento de revoluciones fue muy alta, también lo fue la pérdida de potencia; brindando un índice de eficiencia relativamente bajo (0.11). En cambio, la transmisión de dos pasos tuvo una relación de eficiencia de 1.0, demostrando que su funcionamiento no incrementaba ni disminuía las rotaciones con respecto a la capacidad inicial de la turbina.

\subsection{Selección del generador eléctrico}

Se identificó e implementó un aparato de bajo costo y fácil adquisición, cuyas características debían cumplir con el requisito de generar

Tabla 4. Ventaja mecánica de usar los dos tipos de transmisiones

\begin{tabular}{|l|c|c|c|}
\hline & $\begin{array}{c}\text { Incremento } \\
\text { velocidad }\end{array}$ & $\begin{array}{c}\text { Pérdida de } \\
\text { potencia }\end{array}$ & $\begin{array}{c}\text { Relación de } \\
\text { eficiencia }\end{array}$ \\
\hline Transmisión de tres pasos & $444 \%$ & $77.5 \%$ & $11 \%$ \\
\hline Transmisión de dos pasos & $267 \%$ & $62 \%$ & $100 \%$ \\
\hline
\end{tabular}

electricidad al ser accionado por la turbina de viento.

En un primer momento se experimentó con un alternador de automóvil, el cual genera medianas cantidades de electricidad. Sin embargo, no resultó eficiente, debido a que necesitaba una corriente inicial para inducir el campo magnético que convierte el embobinado interno del rotor en un imán generador. Este proceso, en cierta medida, representó cierto sacrificio de electricidad; aunado al riesgo de que el intenso campo magnético que produce, ejerciera resistencia al movimiento. Esto último provocaría que la turbina no tuviera la fuerza suficiente para accionarlo a niveles productivos.
Dadas estas limitaciones, se optó por utilizar una solución que se ha popularizado a través del Internet, que es sustituir el embobinado interno del rotor por un par de imanes circulares de forma permanente.

Este procedimiento busca generar un campo magnético que no requiera consumo de electricidad y que, por ser de baja potencia, tampoco ofrezca mucha resistencia a la turbina. Sin embargo, dicha solución es ficticia, ya que los dientes del rotor (ver figura 13) se polarizan magnéticamente; $y$ como se ubican en zigzag, dan origen a varios campos opuestos que se anulan entre sí, volviendo imposible la inducción electromagnética. 


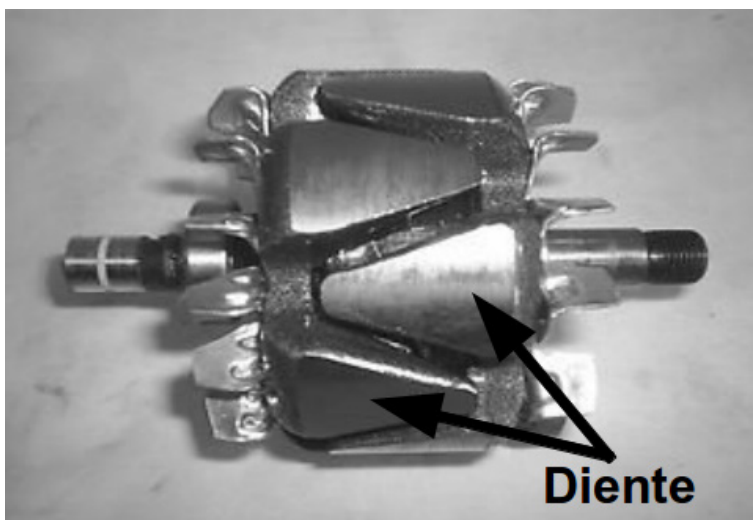

Figura 13. Rotor de alternador. Se visualizan los dientes polarizados magnéticamente (2014).

Otra alternativa difundida en Internet, pero que sí es funcional, es sustituir los dientes del rotor por imanes de neodimio (Figura 14), intercalando la orientación de cada imán para producir inversiones de campo magnético de norte $(\mathrm{N})$ a sur $(\mathrm{S})$; por lo que el fenómeno de inducción de corriente eléctrica se hace posible. La limitante de esta modificación es que su elaboración es más costosa, debido a que cada imán debe incrustarse y asegurarse fuertemente a la estructura del rotor. Además, ese tipo de imánes no se encuentran a la venta en el país, y para utilizarse deben importarse.

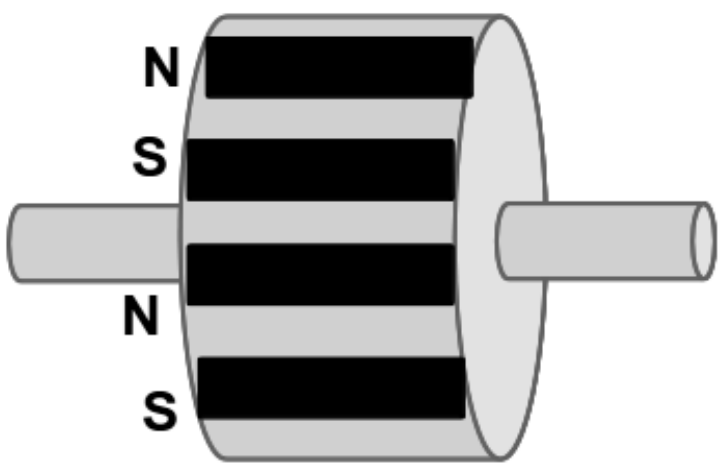

Figura 14. Ejemplificación de rotor con imanes de neodimio incrustados (2014).
Luego de experimentar y descartar la viabilidad de diversas configuraciones del alternador, se optó por buscar una alternativa de corriente continua. Para se adoptó por un motor directo tipo DC, con imanes permanentes, de uso automotriz y de bajas revoluciones (Figura 13), que opera normalmente a 12 voltios y 3 amperios.

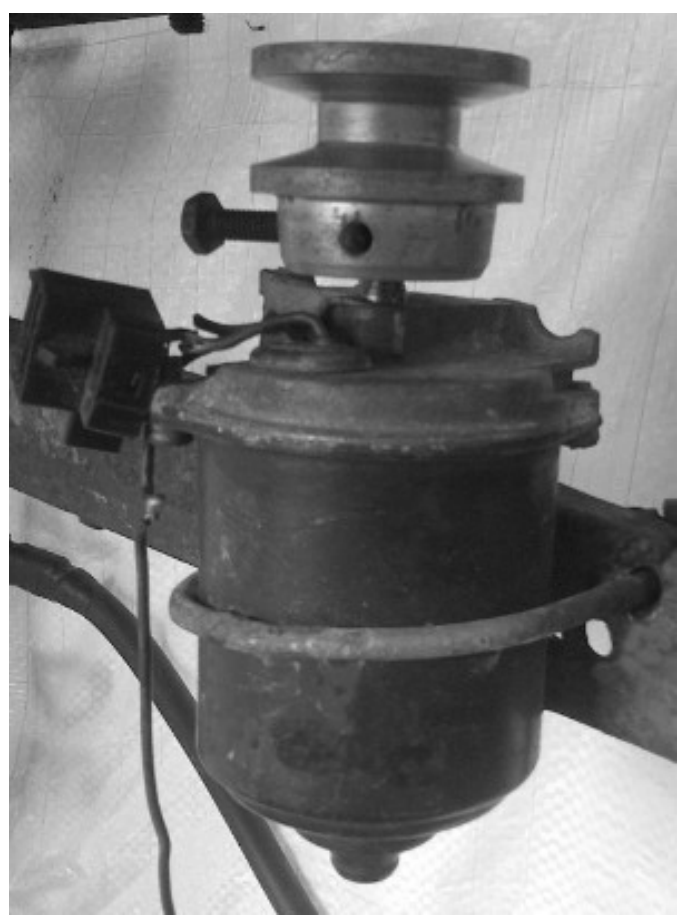

Figura 15. Muestra de un generador de corriente directa (2014).

La potencia normal de este generador está dada por la ley de Watt:

Potencia $(\mathrm{W})=$ Voltaje $(\mathrm{V}) \times$ Corriente $(\mathrm{I})$

Mediante esta ecuación fue posible determinar que la potencia de trabajo es de 36 Watts, cuando las condiciones de operación son estándar. La siguiente gráfica muestra el 
comportamiento de la potencia eléctrica para diferentes valores de corriente.

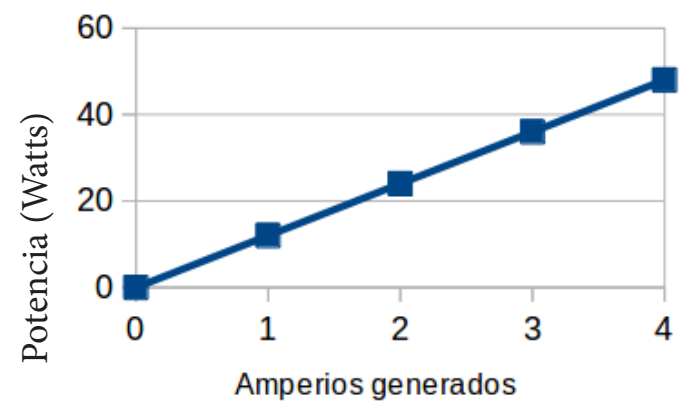

Amperios generados

Figura 16. Gráfico que muestra la relación de potencia según variaciones de corriente.
Debido a que el motor de corriente directa ofrece poca resistencia al movimiento y una capacidad de generación de energía a bajas revoluciones, fue seleccionado como mejor alternativa para el sistema eólico.

\subsection{Producción energética del sistema completo}

Mediante el uso de un multímetro digital y la inducción de corrientes de aire a velocidades controlables, fue posible recoger datos del comportamiento del sistema completo. Se determinaron nuevamente las revoluciones por minuto accionadas por el viento, midiendo el voltaje generado y su respectiva potencia eléctrica.

Tabla 5. Parámetros de producción del sistema completo

\begin{tabular}{|c|c|c|c|}
\hline Viento $(\mathbf{K m} / \mathbf{h})$ & RPM $^{(4)}$ & Voltaje (V) & Watts $(\mathbf{W})$ \\
\hline 5 & 20 & 1.1 & 3.3 \\
\hline 20 & 40 & 2.6 & 7.8 \\
\hline 30 & 82 & 4.3 & 12.9 \\
\hline 40 & 156 & 5.3 & 15.9 \\
\hline
\end{tabular}

Nota: Se observa el resumen de valores arrojados mediante las pruebas a diferente velocidad.

(4) Entiéndase por revoluciones por minuto.

En la siguiente gráfica se representa la tendencia de las revoluciones por minuto accionadas por el viento en el sistema completo. Es evidente el descenso en las rotaciones, comparado con las mediciones obtenidas de la turbina sola (ver figura 8). La razón por la que ocurre esto es porque el generador ejerce resistencia al movimiento del rotor, debido a la fuerza que ejercen sus campos magnéticos internos. Esto da origen a un gasto aproximado de dos tercios de toda la energía mecánica del sistema.

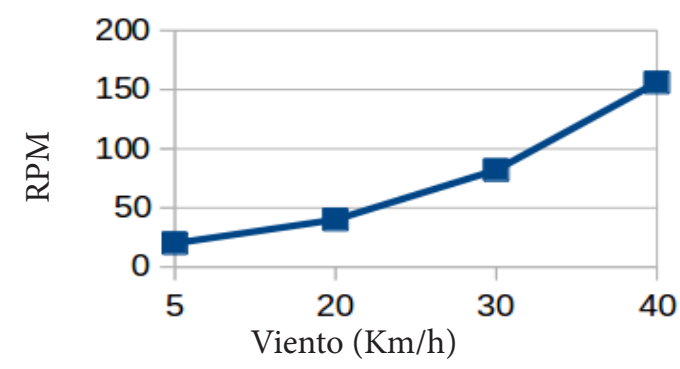

Figura 17. Gráfica que muestra las revoluciones por minuto (rpm) del sistema completo.

En la siguiente figura se modela la tendencia del voltaje generado cuando la turbina opera a diferentes revoluciones por minuto. 


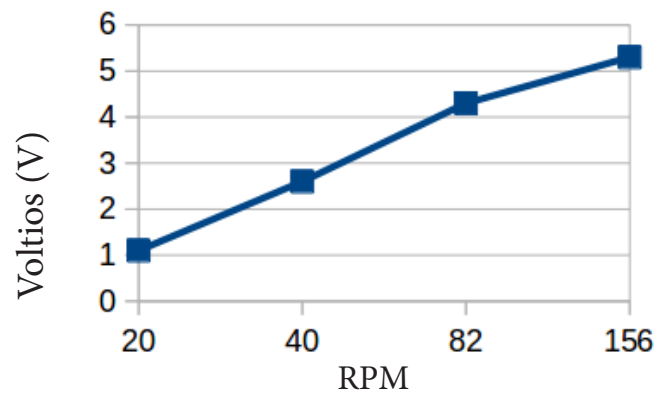

Figura 16. Gráfica que muestra la producción de energía (voltios) del sistema completo. Se observan las cifras expresadas en revoluciones por minuto (rpm).

\subsection{Costos de fabricación}

A continuación se detallan los costos de fabricación de la turbina generadora utilizada en el estudio, que pueden ser tomados como referencia para futuras investigaciones.

Estas cifras no incluyen la totalidad de materiales utilizados durante todo el proceso de experimentación, ni los desembolsos realizados para realizar la investigación; sino solo aquellos que forman parte del producto final que se consideró funcional.

Tabla 6. Costos de fabricación de una aeroturbina generadora

\begin{tabular}{|c|c|c|c|c|}
\hline $\mathrm{Ka}$ & Elemento & $\begin{array}{l}\text { Cantidad } \\
\text { utilixada }\end{array}$ & $\begin{array}{l}\text { Pretio } \\
\text { unitario }\end{array}$ & Conto total \\
\hline 1 & Angular de hierro de $1^{\prime}$ & 4 & 5725 & 379.00 \\
\hline 2 & Varlla plasa 1" & 4 & $\$ 400$ & 316.00 \\
\hline 3 & Tube de hiemo de 3 & 1 & $\$ 2200$ & 327.00 \\
\hline 4 & Bulenon & 2 & $\$ 1500$ & 830.00 \\
\hline 5 & Chamscra & 2 & $\$ 1800$ & 336.00 \\
\hline 6 & Polena" & 1 & $\$ 3800$ & 339.00 \\
\hline$s$ & Poles 5 & ] & 5800 & 8.00 \\
\hline 10 & Imanes Fermanentes & 2 & 530 & MoO \\
\hline II & Bues metilis & ] & $\$ 6000$ & SODO0 \\
\hline 12 & Tuto PrCs $\mathrm{x} 6 \mathrm{~m}$ & I & $\$ 5200$ & 592,00 \\
\hline It & Faja de trangm & I & $\$ 1200$ & 512,00 \\
\hline 16 & Motor DA 12 witios & I & $\$ 3000$ & $\$ 900$ \\
\hline 18 & Servido de tomo & 2 & 5600 & 512000 \\
\hline 19 & Servido de soldatura & I & 55000 & 50000 \\
\hline 30 & Rit de pemos & 1 & $\$ 1200$ & $\$ 12.00$ \\
\hline 27 & Acople de generador & 1 & 5300 & 50,00 \\
\hline 24 & pliego de plywood & I & $\$ 1200$ & 512,00 \\
\hline 25 & Ingtalucion de woletor & I & $\$ 5000$ & 50000 \\
\hline \multicolumn{4}{|c|}{ TGTAL } & $\$ 593.00$ \\
\hline
\end{tabular}

Nota: Cabe señalar que los precios fueron cotizados durante el tiempo de realización de la investigación (año 2014). Las cifras pueden variar acorde a vigencia del producto en el mercado y/o país de utilización. 


\section{Conclusiones}

El diseño de la turbina demostró ser eficiente, capaz de operar a partir de vientos suaves de $5 \mathrm{Km} / \mathrm{h}$, independientemente de su dirección y de la turbulencia que los acompañe. Ante vientos fuertes, es decir arriba de $40 \mathrm{Km} / \mathrm{h}$, la turbina fue capaz de experimentar más de 400 revoluciones por minuto, lo que resultó relativamente satisfactorio.

La fabricación de la turbina fue sencilla, ya que solo necesitó de una estructura metálica que le sirviera de base; y de las palas o alerones de viento fabricados a partir de material PVC. Otra ventaja de este diseño fue su total sigilo para trabajar en todo momento.

Para este diseño de aeroturbina no es necesario incorporar una transmisión que incremente las revoluciones por minuto. Esto debido a que la energía utilizada en incrementar la velocidad se pierde en el esfuerzo giratorio. Por ello se necesitan velocidades de viento mayores para hacer operar el sistema a mayor potencia.

Al experimentar con diferentes diseños de transmisiones, el modelo más eficiente que se encontró equilibraba las rotaciones extra obtenidas con el aumento en la demanda de la velocidad de viento. Gracias a esta relación de equilibrio en la energía mecánica, el generador eléctrico funcionó de igual manera al estar conectado o desconectado de la transmisión.
Un factor importante fue el cambio climático que afecta tanto a El Salvador como a otros países y de diversas maneras. Un ejemplo claro es la sequía que abarcó del 6 de julio al 4 de agosto de 2014, en la cual las condiciones climáticas fueron tan estables, que ni siquiera se produjeron brisas que operaran la turbina a niveles productivos para la generación de energía. Durante ese mismo año, los tradicionales vientos que ocurren en el mes de octubre no se manifestaron hasta la segunda quincena del mes de noviembre. Todos esos factores impactaron de forma negativa en la viabilidad técnica de incursionar en energía eólica a nivel doméstico en el país.

De acuerdo a las observaciones, se pudo determinar que los colectores de viento ofecieron un aumento no mayor al 15\% de las rotaciones de la turbina. Ello es un beneficio muy bajo en virtud de los costos relativos de implementación de la misma.

Al analizar el impacto y desempeño de los generadores, se pudo observar que los generadores de corriente continua DC son los más apropiados para las aplicaciones de energía eólica, dadas las bajas potencias que requieen, por parte del viento, para producir electricidad.

Por su parte, los generadores de corriente alterna AC necesitan mayor potencia para poder trabajar. Pero al realiza la modificación de su estructura interna con imanes 
permanentes para mejorar su rendimiento; si bien su costo incrementa significativamente, su producción energética no lo hace.

Al comparar estos datos, es evidente que la corriente directa DC es mucho más conveniente para la generación eólica, dado que esta puede ser almacenada directamente en una batería o ser usada en forma inmediata con dispositivos de iluminación de bajo consumo (led). En cambio, la corriente alterna AC necesita del diseño e implementación de un circuito rectificador que la convierta sus impulso eléctricos en DC para hacerla aprovechable.

\section{Referencias}

Antezana, J.C. (2004). Diseño y construcción de un prototipo de generador eólico de eje vertical. Universidad de Chile. Recuperado de: http://www.centroenergia.cl/literatura/memorias_tesis/ AEROGENERA DOR-MEMORIA-FINAL.pdf

Ayala, M. y otros. (2005). Determinación del potencial solar y eólico en El Salvador. Ministerio de Medio Ambiente y Recursos naturales (MARN), Universidad Centroamericana "José Simeón Cañas" (UCA), Servicio Nacional de Estudios Territoriales (SNET/MARN). Recuperado de: http://cef.uca.edu.sv/descargables/ proyectos/UCASolarAssessment_es_206.pdf

Berdugo, O.; Suárez, J. (2011). Diseño y construcción de un prototipo de turbina eólica de eje vertical para generación a baja potencia. Universidad Industrial de Santander. Recuperado de: http://tangara.uis.edu.co/biblioweb/tesis/2011/137798. pdf

Bravo, R.; Tullis, S.; Ziada, S. (2007). Pruebas de rendimiento de una aeroturbina de eje vertical pequeña. Universidad McMaster, Estados Unidos. Recuperado de: http://www.eng.mcmaster. ca/ stullis/index_files/Bravo\%20CANCAM\%202007.pdf

Carrera, E.; Collaguazo, P. (2013). Diseño Y Construcción De Un Dispositivo Aerogenerador De Eje Vertical Con Capacidad Mínima De 500 Vatios De Energía Eléctrica. Universidad Politécnica Salesiana, Sede Quito, Ecuador. Recuperado de: http://www.dspace.ups.edu.ec/ bitstream/123456789/6009/1/UPS-KT00203.pdf

Covas, E. A. (2007). La energía eólica en la República Argentina: Aportes para el desarrollo de su aprovechamiento masivo. Universidad Nacional de La Plata, Argentina. Recuperado de: http://www.cadieel.org.ar/ FILES/Contenido1106_1.doc 
Damon, L. (2013). Simulación computarizada de aeroturbinas de eje vertical e hidroturbinas con aspas de inclinación variable. Universidad Boise State, Estados Unidos. Recuperado de: http://scholarworks.boisestate.edu/cgi/viewcontent.cgi? article=1614\&context=td

Freedman, Y.; Zemansky, S. (2009). Física Universitaria. Pearson Education de México. (12a ed.), ISBN 978-1-4244-2291-3.

Gómez, C.; Silió, D. (2007). Aeroturbina lenta de eje vertical con orientación constante de aspa. Universidad de Cantabria, España. Recuperado de: http://bucserver01.unican.es:8080/xmlui/ bitstream/handle/ 10902/1032/ES2269001A1.pdf?sequence=1

Simao, C. y otros. (2007). Análisis de simulación dinámica bidimensional de una aeroturbina de eje vertical: Estrategia de Modelado, verificación y validación de datos de velocimetría de imágenes de partículas. Journal of Physics: Conference Series 75. Recuperado de: http:// iopscience.iop.org/1742-6596/75/1/012023/pdf/ 1742 -6596_75_1_012023.pdf

Simao, C. (2009). La aproximación estelar de la aeroturbina de eje vertical: análisis aerodinámico bidimensional y tridimensional. Wöhrmann Print Service, Zutphen, Países Bajos. Recuperado de: http://repository.tudelft.nl/assets/uuid:ff6eaf63-ac57-492e-a680-c7a50cf5c1cf/ thesis_ library_version.pdf

Sutherland, H. J.; Berg, D.; Ashwill, T. (2012). Una restrospectiva de la tecnología de aeroturbinas de eje vertical. Sandia National Laboratories. Nuevo México, Estados Unidos de América. Recuperado de: http://energy.sandia.gov/wp/wp-content/gallery/uploads/SAND2012-0304. Pdf

Suzuki, M. (2008). Sistema experimental de aeroturbinas de eje vertical con aspas tipo Bellshion. Corporación de Energía Global, Japón. Recuperado de: http://www.jeccomposites.com/news/ composites-news/experimental-vertical-axis-wind-turbine-system-bellshion-blades

Tamayo, J. (2013). Optimización aerodinámica y estructural de un generador eólico de eje vertical. Universidad Pontificia Bolivariana, Colombia. Recuperado de: http://kosmos.upb.edu. co/web/uploads/articulos/\%28A\%29_Optimizacion_Aerodinamica_y _Estructural_de_un_ Generador_Eolico_de_Eje_Vertical_1097.pdf 